\title{
Serum uric acid and renal survival prognosis in primary glomerulonephritis patients in a retrospective single- center cohort
}

\author{
Natalia Stepanova $^{* \mathbb{D}}$, Lyudmila Snisar ${ }^{\mathbb{D}}$, Larysa Lebid ${ }^{\mathbb{D}}$, Svitlana Savchenko, Valentyn Nepomnyashchii, \\ Mykola Kolesnyk \\ State Institution “Institute of Nephrology of the National Academy of Medical Sciences”, Kyiv, Ukraine
}

\section{A R T I C L E I N F O}

Article Type:

Original

\section{Article History:}

Received: 17 July 2020

Accepted: 2 October 2020

Published online: 14 October 2020

Keywords:

Primary glomerulonephritis

Serum uric acid

Estimated glomerular filtration rate

Proteinuria

Renal survival prognosis

End-stage renal disease

\begin{abstract}
A B S T R A C T
Introduction: The role of serum uric acid (SUA) concentration in primary glomerulonephritis (PGN) aggravation is currently under active discussion.

Objectives: This study primarily aimed to analyze the association between SUA concentration and renal survival prognosis in PGN patients and secondarily to determine whether hyperuricemia is an independent risk factor for reduced glomerular filtration rate (GFR) in the presence of nephrotic syndrome.

Patients and Methods: We performed a retrospective observational single-center study involving 344 patients with biopsy-proved or clinically diagnosed PGN with the mean followup period of 5.3 [3.8-6.2] years. The rate of annual decline in estimated GFR (eGFR) was used to assess chronic kidney disease progression. Primary outcome measures were eGFR decline or transfer to renal replacement therapy (RRT) during the 5-year follow-up period.

Results: There were 78/344 (22.7\%) patients who eventually progressed to eGFR $<15 \mathrm{~mL} /$ $\mathrm{min} / 1.73 \mathrm{~m}^{2}$ or started RRT. In multivariate logistic regression analysis eGFR at diagnosis, proteinuria and hyperuricemia were associated with increased renal risk in PGN patients during the 5-year follow-up period. However, a less significant effect of SUA on rapid eGFR decline was found in the patients with nephrotic-range proteinuria compared with the patients with mild proteinuria.

Conclusion: Our study revealed that a higher level of SUA was significantly associated with a greater annual decline in GFR and, consequently, a worse 5-year renal survival prognosis in PGN patients. The effect of hyperuricemia on the risk of rapid CKD progression was greater in PGN patients with mild proteinuria compared with the patients with nephrotic-range proteinuria.
\end{abstract}

\section{Implication for health policy/practice/research/medical education:}

The central research questions raised in the present study were: 1) whether the association between SUA concentration and renal survival prognosis really existed and 2) whether hyperuricemia was an independent risk factor for GFR (glomerular filtration rate) reduction in the presence of nephrotic syndrome. The main finding of our study was the strong association between hyperuricemia and rapid renal function decline in primary glomerulonephritis patients. Interestingly, a 4 -fold greater effect of high SUA concentration on CKD progression was detected in the patients with the mild proteinuria compared with the patients with nephrotic-range proteinuria.

Please cite this paper as: Stepanova N, Snisar L, Lebid L, Savchenko S, Nepomnyashchii V, Kolesnyk M. Serum uric acid and renal survival prognosis in primary glomerulonephritis patients in a retrospective single-center cohort. J Renal Inj Prev. 2021; 10(2): e11. doi: 10.34172/jrip.2021.11.

\section{Introduction}

Primary glomerulonephritis (PGN) is one of the leading causes of end-stage renal disease (ESRD) worldwide (1). According to the European Renal Association-European Dialysis and Transplant Association Registry Annual Report (2016), PGN varies from $16.1 \%$ to $32.5 \%$ in ESRD structure depending on the country (2). Preserving renal function is one of the most important goals in the management of patients with CKD in general and PGN in particular. The major risk factors including age, hypertension, higher proteinuria level and renal function at diagnosis have been suggested as strong 
predictors for ESRD development in PGN patients (3). In addition to these classical risk factors, hyperuricemia has been reported to be an independent risk factor for the development and progression of $\operatorname{CKD}(4,5)$.

The role of serum uric acid (SUA) concentration in chronic kidney disease (CKD) aggravation is currently under active discussion and reevaluation. Evidence of the association between hyperuricemia and a decline in estimated glomerular filtration rate (eGFR) has been accumulated from numerous epidemiological and clinical observations $(4,6,7)$. While a significant number of studies have indicated that a higher level of SUA concentration is associated with a decline in eGFR in the CKD population $(4,5,7)$, the other researchers have found no association between hyperuricemia and a decline in renal function or time to renal replacement therapy (RRT) initiation (8). Additionally, only a few studies have investigated the effect of hyperuricemia on kidney function in PGN patients. However, most of these studies have focused on immunoglobulin A nephropathy (IgAN) (9). In addition, the general cohort of PGN patients was evaluated in the only research (10).

Recent studies suggest a direct correlation between hyperuricemia and proteinuria $(4,10)$. However, the association between hyperuricemia and proteinuria in PGN patients with nephrotic-range proteinuria is still unknown.

\section{Objectives}

The present study primarily aimed to analyze the association between SUA concentration and renal survival prognosis in the patients with a clinical diagnosis of PGN during the 5-year follow-up period and secondarily to determine whether hyperuricemia is an independent risk factor for reduced eGFR in the presence of nephrotic syndrome.

\section{Patients and Methods \\ Study design}

This retrospective observational single-center study was a part of an ongoing Institute's project "Effect of oxalate and urate metabolism on the evolution of kidney disease" (ClinicalTrials.gov Identifier: NCT04399915, Domestic Trial Registration Number: 0117U002122).

\section{Study population}

As presented in Figure 1, the clinical data were retrospectively collected from 575 patient medical records at the primary diagnosis of PGN from 2011 to 2013. Subsequently, the participants with follow-up visit reports or transfer to renal replacement therapy (RRT) during the 5 -year follow-up period were selected, whichever occurred first. The inclusion criteria were; 1) patients older than 18 years; 2) biopsy-proved or clinically diagnosed objects with PGN; 3) outpatient follow-up data included at least 3 serial SUA and creatinine concentrations during the

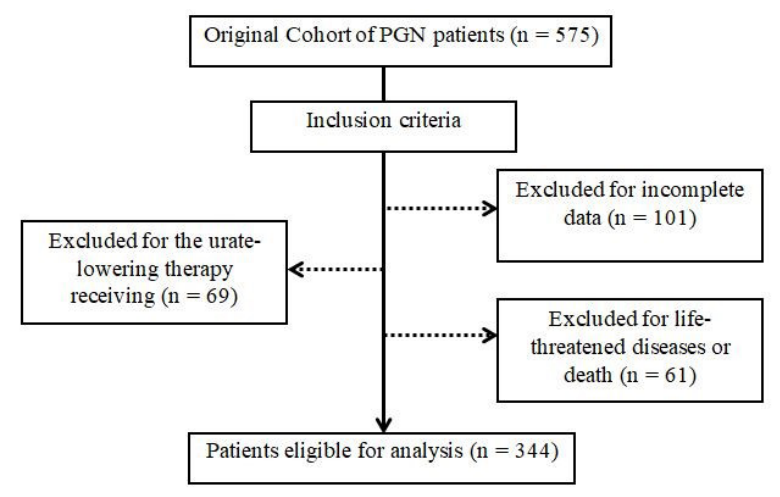

Figure 1. Flow diagram of the study population.

5-year follow-up period; 4) eGFR was $\geq 30 \mathrm{~mL} / \mathrm{min} / 1.73$ $\mathrm{m}^{2}$.

We further excluded 231 participants from 575 PGN patients; the participants with incomplete information 101, the subjects received urate-lowering therapy - 69, the patients with a history of the life-threatened disease which could affect CKD progression (acute myocardial infarction, stroke, surgery due to any reasons, etc.) - 58, 3 patients died.

Thus, a total of 344 patients were included in this study. Among them, there were 194 (56.4\%) patients with biopsy-proven PGN and 150 (43.6\%) patients with a clinical diagnosis of PGN. All patients were treated according to KDIGO Clinical Practice Guidelines for Glomerulonephritis (11) and agreements.

\section{Measurements and data collection}

The baseline characteristics of the participants included age, gender and laboratory measurements. All clinical parameters were measured at the time of a patient's first clinic visit or renal biopsy.

SUA levels, blood creatinine $(\mathrm{Cr})$ concentration, urea (Ur), albumin, total cholesterol (TC), fasting blood glucose, hemoglobin $(\mathrm{Hb}), \mathrm{C}$-reactive protein (CRP), serum electrolytes and daily proteinuria levels were collected. All routine biochemical parameters were carried out using a Flexor Junior Chemistry Analyzer (Vital Scientific, Dieren, the Netherlands). Hematological parameters of blood were determined using an "ABX Micros-60" (France). Hematological parameters of blood were determined using an ABX Micros-60 (ABX Diagnostics, Montpelier, France). Urine protein excretion (UPE) was measured in a $24-\mathrm{h}$ urine collection.

eGFR (milliliters per minute per $1.73 \mathrm{~m}^{2}$ ) was calculated using the Chronic Kidney Disease Epidemiology Collaboration (CKD-EPI) formula and its baseline value was based on the first available eGFR in PGN diagnosed patients. None of the patients was on urate- or lipidlowering therapy at the time of baseline data.

Ultrasound-guided renal biopsy was obtained after taking the written informed consent from the patients 
and excluding contraindications. The histopathological evaluation was performed by one renal pathologist blinded to the clinical data.

\section{Clinical definition}

Hyperuricemia was defined as SUA concentration $\geq 420 \mu \mathrm{mol} / \mathrm{L}(7 \mathrm{mg} / \mathrm{dL})$ in male and $\geq 360 \mu \mathrm{mol} / \mathrm{L}(6 \mathrm{mg} /$ $\mathrm{dL}$ ) in female. Arterial hypertension $(\mathrm{AH})$ was defined as systolic blood pressure $\geq 140 \mathrm{~mm} \mathrm{Hg}$ and diastolic blood pressure $\geq 90 \mathrm{~mm} \mathrm{Hg}$.

The annual eGFR decline rate was used to assess CKD progression. It was calculated by the difference between eGFR $\left(\mathrm{mL} / \mathrm{min} / 1.73 \mathrm{~m}^{2}\right)$ at baseline and the values: (Last eGFR - Baseline eGFR)/Follow-up period per year). Nephrotic-range proteinuria was defined as urinary protein excretion $\geq 3.5 \mathrm{~g} / \mathrm{d}$.

\section{Ethical issues}

The research followed the tenets of the Declaration of Helsinki. The study protocol was confirmed by the Ethics Committee of the Institute (Protocol 1/19 from January $11,2019)$ and writing informed consent was obtained from all the subjects participating in the study. The study was a part of an ongoing Institute project: "Effect of oxalate and urate metabolism on the evolution of kidney disease" (ClinicalTrials.gov, identifier: NCT04399915, Domestic Trial Registration Number: 0117U002122).

\section{Statistical analysis}

The statistical analysis and all graphs were performed using MedCalc (Belgium). The average means (M), the standard deviations (SD) or the median $(\mathrm{Me})$ and the interquartile ranges [Q25 - Q75] were calculated according to the distribution. For the statistical analysis, we used the Student's $t$ test and the nonparametric (U-test) MannWhitney. The Mann-Whitney U or the Kruskal-Wallis tests (as appropriate) were used to compare non-normally distributed continuous variables.

Categorical variables were expressed as proportions, and, the chi-square tests were used to compare 2 groups. Pearson's or Spearman's correlation tests (as appropriate) were used to evaluate the association between SUA and other parameters. Univariate and multiple logistic regression analyses were fitted to assess the factors affecting increased progression of CKD. The strength of the association between SUA and CKD progression was expressed as odds ratio (OR) and 95\% confidence interval (CI). The Cox proportional-hazard multiple regression model was used to determine a cumulative 5-year renal survival prognosis. $P$ values were calculated, and, the null hypothesis was rejected if the $P$ value was $<0.05$.

\section{Results}

Patient characteristics

A total of 344 patients with PGN were observed with the mean follow-up period of 5.3 [3.8-6.2] years. Among them, there were $206(60 \%)$ men and 138 (40\%) women $(P=0.15)$. The patients' age varied from 18 to 78 years and averaged 41 [31-56] years. Among renal biopsy findings, there were membranous GN (64/194, 32\%), focal segmental glomerular sclerosis 'NOS' (42/194, 21.7\%) and 'tip' (34/194, 17.5\%), minimal change disease (33/194, $13.5 \%)$ and $\operatorname{IgA}$ nephropathy $(21 / 194,10.8 \%)$.

Hyperuricemia was found in 72/206 (35\%) men and $38 / 138(27.5 \%)$ women $(P=0.14)$, and an increasing trend in SUA was significantly corresponded with the progression of CKD stages (Figure 2).

For the analysis, the patients were gender-stratified into the quartiles according to average SUA levels at baseline: Q1: $<265 \mu \mathrm{mol} / \mathrm{L}$ for men and $<220 \mu \mathrm{mol} / \mathrm{L}$ for women, Q2: $265-446 \mu \mathrm{mol} / \mathrm{L}$ for men and 220-369 $\mu \mathrm{mol} / \mathrm{L}$ for women, Q3: $\geq 447 \mu \mathrm{mol} / \mathrm{L}$ for men and $\geq 370$ for women, respectively (Figure 3 ).

The baseline characteristics of the SUA groups stratified into the quartiles are shown in Table 1.

As presented in Table 1, there was no difference in

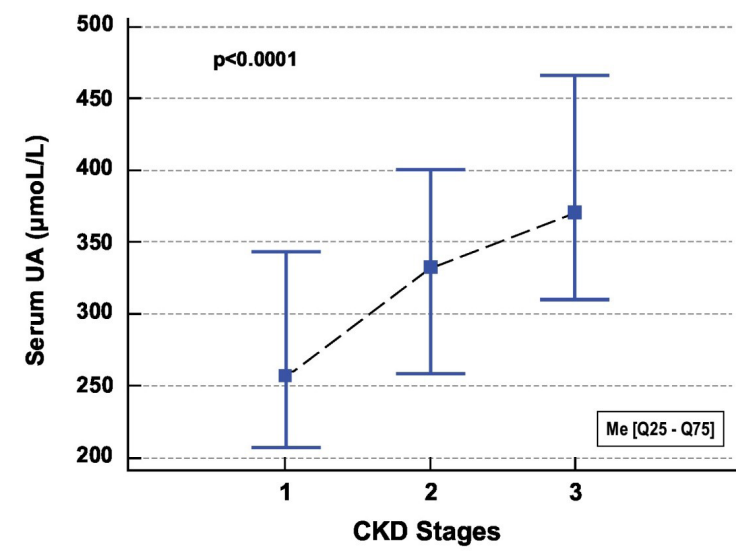

Figure 2. The distribution of baseline SUA levels according to CKD stages (The Kruskal-Wallis test).

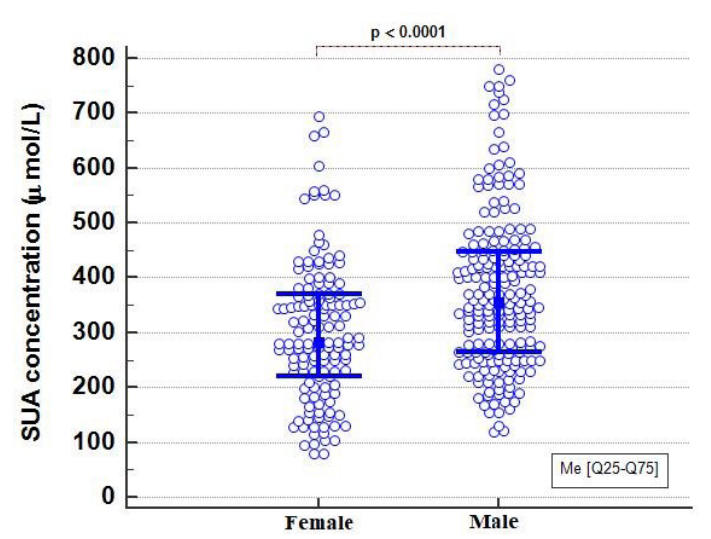

Figure 3. The gender-based distribution of SUA concentrations at baseline. 
Table 1. Characteristics of the study groups stratified into SUA quartiles

\begin{tabular}{|c|c|c|c|c|c|}
\hline Parameter, unit & All $(n=344)$ & Q1 ( $n=84)$ & Q2 (n = 165) & Q3 (n = 95) & $P$ \\
\hline Male gender, n (\%) & 206 (60\%) & $40(47.6 \%)^{*}$ & $95(57.6 \%)^{*}$ & $71(74.8 \%)^{*}$ & 0.0004 \\
\hline Age, years & $41[29-54]$ & 35 [26-47.5] & 43 [29.3-55] & $40[29-56]$ & 0.08 \\
\hline Follow-up period, years & $5.3[3.8-6.2]$ & $5.4[2.8-6.6]$ & $5.2[2.7-6.2]$ & $4.7[2.1-5.5]$ & 0.46 \\
\hline \multicolumn{6}{|l|}{ Clinical parameters } \\
\hline Serum albumin, $\mathrm{g} / \mathrm{L}$ & 35.8 [29.8-45.6] & $36.8[30-44]$ & $33[29-40.5]^{*}$ & $39.1[32.5-43.5]^{*}$ & 0.017 \\
\hline Blood protein, g/L & 58 [50.4-65] & 58.3 [49.3-68.3] & $56[49.5-62.9]^{*}$ & $61.5[54.5-64.9]^{*}$ & 0.02 \\
\hline UPE, g/d & $3.57[1.4-6.7]$ & $4[1.5-6.6]$ & $3.8[1.2-7.2]$ & $2.9[1.4-6.4]$ & 0.71 \\
\hline $\mathrm{Cr}, \mu \mathrm{mol} / \mathrm{L}$ & 107.3 [87-167.5] & $90.8[75.4-112.8]^{*}$ & $103[86.4-167]^{*}$ & 134 [101-198.8]* & 0.0001 \\
\hline Ur, $\mathrm{mmol} / \mathrm{L}$ & $6.95[4.7-11.3]$ & $5.2[4.2-7.7]^{*}$ & $7.0[4.7-9.9]^{*}$ & $8.9[5.4-19.3]^{*}$ & $<0.0001$ \\
\hline eGFR, $\mathrm{mL} / \mathrm{min} / 1.73 \mathrm{~m}^{2}$ & 73.3 [45-90] & $87.6[69-100]^{*}$ & $76[48.5-90]^{*}$ & $52.8[38-73.5]^{*}$ & $<0.0001$ \\
\hline CRP, mg/L & $6[5.4-7.2]$ & $4[3.6-5]^{*}$ & $6.3[5.4-7.2]^{*}$ & $14.2[9-22.3]^{*}$ & 0.001 \\
\hline $\mathrm{AH}, \mathrm{n}(\%)$ & 306 (89\%) & $68(82 \%)^{*}$ & $143(87 \%)^{*}$ & $95(100 \%)^{*}$ & 0.0003 \\
\hline $\mathrm{Hb}, \mathrm{g} / \mathrm{L}$ & 130 [114-146] & 131 [121-148] & 130 [114-147] & $123[113-140]$ & 0.11 \\
\hline Glucose, $\mathrm{mmol} / \mathrm{L}$ & $5.2[4.7-5.6]$ & $5.2[4.8-5.5]$ & $5.3[4.7-5.6]$ & $5.3[4.7-5.65]$ & 0.74 \\
\hline Calcium, mmol/L & $2.18[2.0-2.33]$ & 2.19 [2.0-2.33] & $2.16[2.0-2.28]$ & $2.2[2.0-2.3]$ & 0.4 \\
\hline Phosphorus, $\mathrm{mmol} / \mathrm{L}$ & $1.15[0.99-1.45]$ & $1.0[0.89-1.2]^{*}$ & $1.12[0.96-1.45]^{*}$ & $1.26[1.05-1.65]^{*}$ & 0.0004 \\
\hline Potassium, mmol/L & $4.68[4.2-5.1]$ & $4.4[4.0-4.9]^{*}$ & $4.7[4.2-5.2]^{*}$ & $5.03[4.4-5.4]^{*}$ & 0.0002 \\
\hline $\mathrm{TC}, \mathrm{mmol} / \mathrm{L}$ & $6.9[5.0-9.1]$ & $6.4[4.8-9.1]$ & $7.8[6.0-9.4]^{*}$ & $6.3[4.5-7.6]^{*}$ & $<0.0001$ \\
\hline \multicolumn{6}{|l|}{ Medications, n (\%) } \\
\hline ACE inhibitors / RAAS blockers & 189 (54.9 \%) & $56(66.6 \%)$ & $107(64.8 \%)^{*}$ & $26(27.4 \%)^{*}$ & $<0.0001$ \\
\hline Diuretics & $168(48.8 \%)$ & $44(52.4 \%)$ & $93(56.4 \%)^{*}$ & $31(32.6 \%)^{*}$ & 0.0002 \\
\hline Lipid-lowering therapy & 66 (19.2\%) & $19(22.6 \%)$ & $29(17.6 \%)$ & $18(19 \%)$ & 0.53 \\
\hline Use of corticosteroids & $82(23.8 \%)$ & $21(25.0 \%)$ & $37(22.4 \%)$ & $24(25.3 \%)$ & 0.26 \\
\hline Use of cytostatics & $133(38.6 \%)$ & $36(42.8 \%)^{*}$ & $69(41.8 \%)$ & $28(29.5 \%)^{*}$ & 0.04 \\
\hline
\end{tabular}

*A statistically significant difference between the groups (the Kruskal-Wallis test).

Abbreviations: ACE inhibitors, angiotensin-converting enzyme inhibitors; AH, arterial hypertension; Cr, creatinine; CRP, C-reactive protein; eGFR, estimated glomerular filtration rate; $\mathrm{Hb}$, hemoglobin; RAAS blockers, renin-angiotensin-aldosterone system blockers; TC, total cholesterol; UPE, urine protein excretion; Ur, urea.

baseline age, a follow-up period, UPE, Hb concentration and blood glucose level among SUA quartiles. However, the patients with the highest SUA level had higher levels of $\mathrm{Cr}$, Ur and potassium with a corresponding decreasing trend in eGFR. This quartile also had a lower prevalence of diuretics and cytostatics users compared with the patients of Q2.

The association between SUA and CKD progression During the 5-year average follow-up period, there were $78 / 344(22.7 \%)$ patients who eventually progressed to eGFR $<15 \mathrm{~mL} / \mathrm{min} / 1.73 \mathrm{~m}^{2}$ or started RRT. Among them, there were: Q1 - $10(12,8 \%)$ patients, Q2 - 25 (32\%) patients and Q3 - $43(55.2 \%)$ patients $(P<0.0001)$. As might be expected, the highest renal progression level was observed in the patients of Q3: $-5.5[-15.4 ;-1.8] \mathrm{mL} /$ $\mathrm{min} / 1.73 \mathrm{~m}^{2}$ vs $-3.5[-6.4 ;-1.7]$ and $-4.6[-10.6 ;-2.7] \mathrm{mL} /$ $\mathrm{min} / 1.73 \mathrm{~m}^{2}$ in the patients of Q2 and Q1, respectively (Figure 4).

However, a low SUA level (Q1) did not demonstrate the effect on increased progression of CKD in univariate logistic regression analysis. eGFR $<60 \mathrm{~mL} / \mathrm{min} / 1.73 \mathrm{~m}^{2}$ at PGN diagnosis, age $\geq 65$ years, hypertension $>140 / 90$ $\mathrm{mm} \mathrm{Hg}, \mathrm{UPE} \geq 3.5 \mathrm{~g} / \mathrm{d}$ and hyperuricemia were strong predictors for a 5-year ESRD development in the PGN patients (Figure 5).

In addition, while univariate analysis demonstrated a 2.5fold [95\% CI 1.5-4.3] increased renal risk in the patients with hyperuricemia, the multivariate analysis showed a 4.4-fold [95\% CI 2.03-10.8] increased renal risk after adjustment for eGFR at PGN diagnosis, age, hypertension

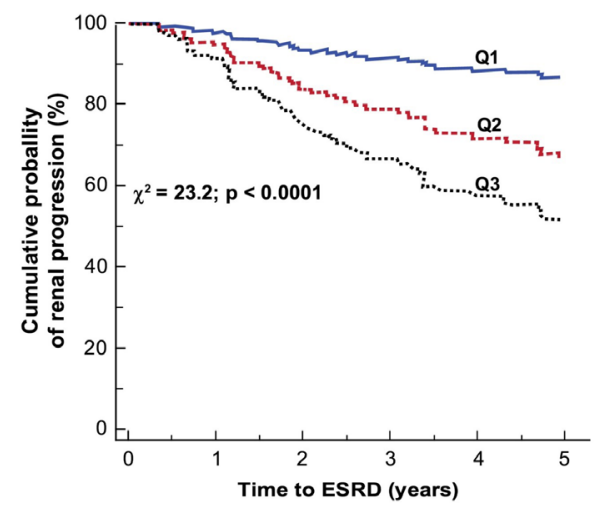

Figure 4. The average annual decline in GFR according to baseline SUA levels in PGN patients. 
and proteinuria level. It is noteworthy mentioning that in addition to hyperuricemia, only eGFR $<60 \mathrm{~mL} / \mathrm{min} / 1.73$ $\mathrm{m}^{2}$ (OR 94 [95\% CI 91.7-276.3], $P<0.0001$ ) and 24-hour proteinuria level (OR 1.1 [95\% CI 1.05-1.3], $P=0.001$ ) were included in the multiple stepwise logistic regression model (overall model fit: $\chi^{2}=173.5, P<0.0001$ ).

Figure 6 demonstrates a better cumulative 5-year renal survival prognosis in the patients of Q1 compared with the patients of Q2 (HR 2.8 [CI 95\% 1.8-2.5]) and Q3 (HR 4.02 [CI 95\% 2.4-4.7]), respectively.

\section{Hyperuricemia and the risk of eGFR decline according to 24-hour proteinuria level}

As presented in Table 1, the UPE level did not differ among SUA quartiles, and, it was not associated with SUA concentration ( $\mathrm{r}=-0.03, P=0.95)$. However, an inverse correlation between UPE and eGFR changes was observed at the end of the follow-up period ( $\mathrm{r}=-0.17, P=0.001)$. For further interaction analysis between proteinuria and SUA, we examined the effect of hyperuricemia on $\mathrm{CKD}$ progression according to the proteinuria level.

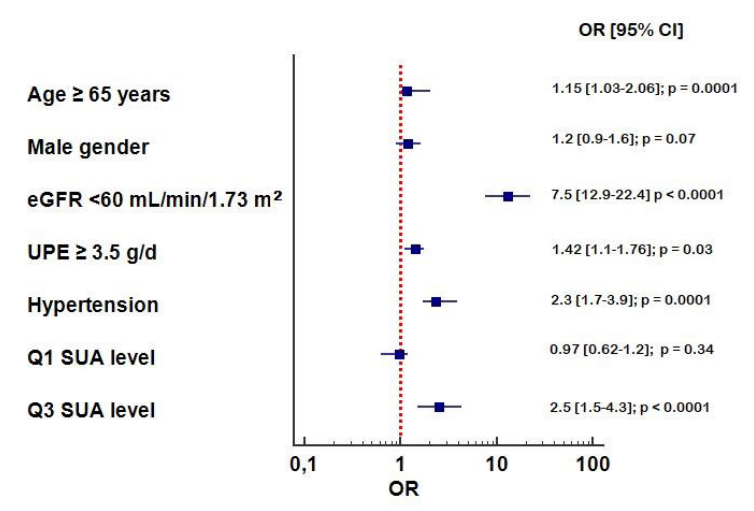

Figure 5. Odds ratio [95\% confidence interval] for ESRD development in the PGN patients (univariate analysis).

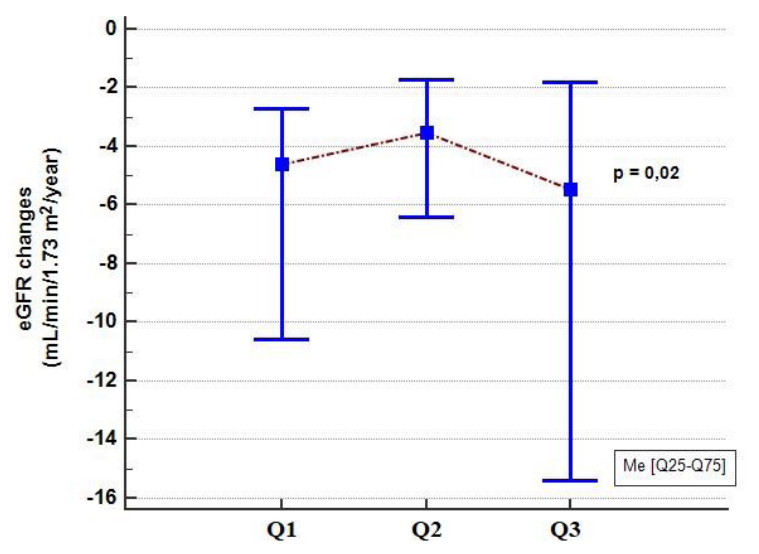

Figure 6. The 5-year cumulative renal survival prognosis stratified by SUA concentration.
The average UPE was $6.3[4.4-10] \mathrm{g} / \mathrm{d}$ in the patients with nephrotic-range proteinuria vs $1.03[0.4-1.9] \mathrm{g} / \mathrm{d}$ in the patients with mild proteinuria $(P<0.0001)$. In multivariate logistic regression analysis for predicting rapid renal function decline, eGFR at PGN diagnosis, proteinuria and hyperuricemia were still associated with rapid renal function decline in the PGN patients during the 5-year follow-up period (overall model fit: $\chi^{2}=34.3$, $P<0.0001)$. However, a less significant effect of SUA on rapid eGFR decline was found in the patients with nephrotic-range proteinuria compared with the patients with mild proteinuria (Table 2).

\section{Discussion}

The first hypothesis for the potential role of SUA in CKD progression was proposed almost a century ago. However, it was rejected too hastily in the early eighties $(12,13)$. In recent years, numerous experimental and clinical studies have been conducted to examine the effect of SUA on eGFR decline $(4,7,9,10)$. Nevertheless, the causal relationship between SUA concentration and CKD progression still remains debatable. High heterogeneity in the studied CKD population, as well as determining rapid CKD progression, does not allow a final conclusion.

The present study focused only on PGN patients, and this criterion ensured the homogeneity of the investigated cohort. The main research questions for this study were; 1) whether the association between SUA concentration and renal survival prognosis really existed; 2) whether hyperuricemia was an independent risk factor for GFR reduction in the presence of nephrotic syndrome. The main finding of our study was the strong association between hyperuricemia and rapid renal function decline in all PGN cohorts. However, a 4-fold greater effect of high SUA concentration on CKD progression was detected in the patients with the mild proteinuria compared with the patients with nephrotic-range proteinuria.

Our findings support a number of previous studies on the association between hyperuricemia and the high risk of CKD progression $(4,7,10)$. However, only a few works

Table 2. The risk factors for rapid CKD progression among the PGN patients according to nephrotic-range proteinuria (multiple stepwise logistic regression model)

\begin{tabular}{lccl}
\hline Variables & OR & 95\% Cl & p \\
\hline \multicolumn{4}{c}{ The patients with nephrotic-range } \\
\hline eGFR at PGN diagnosis & 1.02 & $1.01-1.03$ & 0.0001 \\
24-hour proteinuria level & 1.14 & $1.06-1.2$ & 0.0004 \\
SUA & 1.7 & $1.04-1.9$ & 0.03 \\
\hline \multicolumn{5}{c}{ The patients with mild proteinuria } \\
\hline eGFR at PGN diagnosis & 1.1 & $1.04-1.15$ & 0.0001 \\
SUA & 4.8 & $1.2-18.5$ & 0.02 \\
\hline
\end{tabular}

Abbreviations: OR, odds ratio; $95 \% \mathrm{Cl}, 95 \%$ confidence interval; PGN, primary glomerulonephritis; eGFR, estimated glomerular filtration rate; SUA, serum uric acid. 
examined the effect of hyperuricemia on CKD progression in PGN patients $(9,10)$. It should be emphasized that the above-mentioned investigations were conducted in the general population with CKD. To the best of our knowledge, this is the first study to demonstrate that hyperuricemia is an independent risk factor for rapid eGFR decline in the presence of nephrotic syndrome.

In a recent study, Li et al have demonstrated the negative association between urinary uric acid excretion and albuminuria, which could be an explanation for rapid CKD progression in hyperuricemia condition (14). It has been reported that proteinuria is associated with increased SUA in the general (15), diabetic (16), heart failure (17) and PGN populations (10). However, in contrast to the mentioned results, we did not find the association between SUA and proteinuria levels in the cohort of PGN patients. Tsai et al analyzed a large heterogeneous cohort of CKD patients and noticed that the effect of hyperuricemia on eGFR decline was more prominent in patients without proteinuria compared with patients with proteinuria (4). The scientists have reached the conclusions: 1) proteinuria is the most important predictor for CKD progression in patients with glomerulonephritis; 2) the effect of SUA on CKD progression is less significant in such conditions (4). In accordance with these findings, we have obtained the absolutely identical results and fully support the above hypothesis.

The explanation for a less significant effect of SUA on CKD progression in patients with nephrotic-range proteinuria is complicated by the fact that kidney disease per se can affect both SUA levels and CKD progression. From one point of view, proteinuria is an established risk factor for CKD progression $(14,17)$ and, consequently, can lead to an increase in SUA concentration. Alternatively, accumulating evidence suggests that increased SUA might be a cause for induced oxidative stress, endothelial dysfunction, an increase in inflammatory responses and the formation of proteinuria (18-20). Furthermore, both glomerular proteinuria (20) and hyperuricemia (10) are the risk factors for glomerulosclerosis, tubular and interstitial damage. However, it is doubtless that renal pathological changes in the patients with nephrotic-range proteinuria are one of the most important prognostic predictors for CKD progression and cannot be explained only by the presence of SUA concentration.

\section{Conclusion}

The findings of the current study are in line with the previous data based on the suggestion that hyperuricemia can be considered as a risk factor for CKD progression. Our study revealed that a higher level of SUA was significantly associated with a greater annual decline in GFR and, consequently, a worse 5-year renal survival prognosis in PGN patients. The effect of hyperuricemia on the risk of rapid CKD progression was greater in the patients with mild proteinuria compared with the patients with nephrotic-range proteinuria. Further welldesigned clinical trials are required to determine the effect of hyperuricemia on renal survival prognosis in PGN patients.

\section{Limitations of the study}

The present study has several important limitations. First, bearing in mind a retrospective design of our study performed in a single-center with a relatively small sample size, our findings have to be interpreted only in terms of associations. Second, in spite of the fact that at least 3 serial SUA data were included, we evaluated only the effect of SUA concentration on CKD progression. Third, we did not analyze the association between SUA and renal pathological changes as it needed large scale prospective studies to prove. Finally, possible effects of medications (glucocorticosteroids, cytostatics, ACE-inhibitors, diuretics) on SUA level were not taken into account.

Notwithstanding these limitations, the study has successfully demonstrated that the strong association between hyperuricemia and rapid kidney function decline observed in the present study indicates the potential negative effect of high SUA on renal survival prognosis in PGN patients. Further studies with a larger sample size are needed to confirm our research findings.

\section{Authors' contribution}

NS conceived the presented concept, designed the study, analyzed and interpreted the patient data, and was a major contributor in writing the manuscript. LS and LL collected the data, SS performed the laboratory measurements, VN performed the histopathological study. MK worked out the concept, contributed to the design and research management. All authors participated in preparing the final draft of the manuscript, revised the manuscript and critically evaluated the intellectual contents. They have read and approved the content of the manuscript and confirmed the accuracy or integrity of any part of the paper.

\section{Conflicts of interest}

The authors declare that they have no competing interests. The part of the data was accepted as an abstract for a poster presentation at the 57th ERA-EDTA Congress, 2020.

\section{Ethical considerations}

Ethical issues including plagiarism, double publication, and redundancy have been completely observed by the authors.

\section{Funding/Support}

The study was carried out within the framework of the Institute's research work "Effect of oxalate and urate metabolism on the evolution of kidney disease" (ClinicalTrials.gov Identifier: NCT04399915, Domestic Trial Registration Number: 0117U002122). 


\section{References}

1. Ali AA, Sharif DA, Almukhtar SE, Abd KH, Saleem ZSM, Hughson MD. Incidence of glomerulonephritis and nondiabetic end-stage renal disease in a developing middle-east region near armed conflict. BMC Nephrol. 2018;19:257. doi: 10.1186/s12882-018-1062-7.

2. ERA-EDTA Registry: ERA-EDTA Registry Annual Report 2016. Amsterdam, the Netherlands: Amsterdam UMC, Department of Medical Informatics; 2018.

3. Chembo C L, Marshall MR, Williams LC, Walker RJ, Lynn KL, Irvine J, Pilmore HL. Long-term outcomes for primary glomerulonephritis: New Zealand Glomerulonephritis Study. Nephrology (Carlton). 2015;20:899-907. doi: 10.1111/nep. 12538.

4. Tsai C-W, Lin S-Y, Kuo C-C, Huang C-C. Serum uric acid and progression of kidney disease: a longitudinal analysis and mini-review. PLoS One. 2017;12:e0170393. doi: 10.1371/journal.pone.0170393.

5. Jalal DI, Decker E, Perrenoud L, Nowak KL, Bispham N, Mehta T, et al. Vascular function and uric acid-lowering in stage 3 CKD. J Am Soc Nephrol. 2017; 28(3):943-52. doi: 10.1681/ASN.2016050521.

6. Stepanova N, Burdeyna O, Snisar L. Hyperuricemia is associated with cardiovascular events and all-cause mortality in peritoneal dialysis patients. Nephrol Dial Transplant. 2019;34:i242. doi: 10.1093/ndt/gfz106.FP587

7. Tseng WC, Chen YT, Lin YP, Ou SM, Yang CY, Lin CH, et al. Hyperuricemia predicts an early decline in renal function among older people: a community-based cohort study. Sci Rep. 2019;9:980. doi: 10.1038/s41598-018-37529-z.

8. Chini LSN, Assis LIS, Lugon JR. Relationship between uric acid levels and risk of chronic kidney disease in a retrospective cohort of Brazilian workers. Braz J Med Biol Res. 2017;50:e6048. doi: 10.1590/1414-431X20176048.

9. Nagasawa Y, Yamamoto R, Shoji T, Shinzawa M, Hasuike Y, Nagatoya K, et al. Serum uric acid level predicts progression of IgA nephropathy in females but not in males. PLoS One. 2016;11:e0160828. doi: 10.1371/journal.pone.0160828.

10. Fan S, Zhang P, Wang AY, Wang X, Wang L, Li G, et al. Hyperuricemia and its related histopathological features on renal biopsy. BMC Nephrol. 2019;20:95. doi: 10.1186/ s12882-019-1275-4.

11. Radhakrishnan J, Cattran DC. The KDIGO practice guideline on glomerulonephritis: reading between the (guide) lines-application to the individual patient. Kidney Intern. 2012;82:840-56. doi: 10.1038/ki.2012.280.

12. Chen MY, Zhao CC, Li TT, Zhu Y, Yu TP, Bao YQ, et al. Serum uric acid levels are associated with obesity but not cardio-cerebrovascular events in Chinese inpatients with type 2 diabetes. Sci Rep. 2017;7:40009. doi: 10.1038/ srep40009.

13. Bellomo G. Uric acid and chronic kidney disease: A time to act? World J Nephrol. 2013;2:17-25. doi: 10.5527/wjn. v2.i2.17.

14. Li F, Guo H, Zou J, Chen W, Lu Y, Zhang X, et al. Urinary excretion of uric acid is negatively associated with albuminuria in patients with chronic kidney disease: a cross-sectional study. BMC Nephrol. 2018;19:95. doi: 10.1186/s12882-018-0892-7.

15. Gertler MM, Garn SM, Levine SA. Serum uric acid in relation to age and physique in health and in coronary heart disease. Ann Intern Med. 1951;34:1421-31. doi: 10.7326/0003-4819-34-6-1421.

16. Scheven L, Joosten MM, de Jong PE, Bakker SJ, Gansevoort RT; PREVEND study group. The association of albuminuria with tubular reabsorption of uric acid: results from a general population cohort. J Am Heart Assoc. 2014;3:e000613. doi: 10.1161/jaha.113.000613.

17. Pinelli M, Bindi M, Moroni F, Castiglioni M. Relationship between serum uric acid levels and urinary albumin excretion in patients with heart failure. Acta Cardiol. 2008; 63(2):191-5. doi: 10.2143/AC.63.2.2029527.

18. Stepanova N, Burdeyna O, Driianska V. Uric acid induces intraperitoneal inflammation in peritoneal dialysis patients. Nephrol Dial Transplant. 2019;34:i238. doi: 10.1093/ndt/ gfz106.FP575.

19. Demikhova N, Chernatska O, Mazur T, Bokova S, Rudenko T, Bumeister L, Demikhov O. Markers of cardiovascular complications in patients with type 2 diabetes mellitus and arterial hypertension. BJMS. 2018;17:319-22. doi: 10.3329/ bjms.v17i2.35894.

20. Mariani LH, Martini S, Barisoni L, Canetta PA, Troost JP, Hodgin JB, et al. Interstitial fibrosis scored on whole-slide digital imaging of kidney biopsies is a predictor of outcome in proteinuric glomerulopathies. Nephrol Dial Transplant. 2018;33:310-318. doi: 10.1093/ndt/gfw443.

Copyright (C) 2021 The Author(s); Published by Nickan Research Institute. This is an open-access article distributed under the terms of the Creative Commons Attribution License (http://creativecommons.org/licenses/by/4.0), which permits unrestricted use, distribution, and reproduction in any medium, provided the original work is properly cited. 